\title{
The GlueX Experiment: Search for Gluonic Excitations via Photoproduction at Jefferson Lab
}

\author{
Paul Eugenio* \\ Florida State University \\ on behalf of the GlueX Collaboration \\ E-mail: eugenioefsu.edu
}

\begin{abstract}
Studies of meson spectra via strong decays provide insight regarding QCD at the confinement scale. These studies have led to phenomenological models for QCD such as the constituent quark model. However, QCD allows for a much richer spectrum of meson states which include extra states such as exotics, hybrids, multi-quarks, and glueballs. First discussion of the status of exotic meson searches is given followed by an overview of the progress at Jefferson Lab to double the energy of the machine to $12 \mathrm{GeV}$, which will allow us to access photoproduction of mesons in search for gluonic excited states.
\end{abstract}

Xth Quark Confinement and the Hadron Spectrum $8 Đ 12$ October 2012

TUM Campus Garching, Munich, Germany

\footnotetext{
* Speaker.
} 


\section{Status of Exotic Mesons}

Discoveries of new phenomena in nuclear and particle physics have provided insight into the fundamental constituents of matter. In the past few decades we have seen a new picture emerge in which quarks form the building blocks of nearly all matter. Yet the gluon, which carries the force which binds quarks, can interact with other gluons to form a bound state, or interact as a fundamental constituent of matter along with the quarks. Thus new forms of gluonic or hybrid matter should exist.

Studies of meson spectra via strong decays of hadrons provide insight regarding QCD at the confinement scale. These studies have led to phenomenological models for QCD such as the constituent quark model. However, QCD demands a much richer spectrum of meson states which includes extra states such as gluonic hybrids $(q \bar{q} g)$, multiquarks $(q \bar{q} q \bar{q})$, and glueballs ( $g g$ or $g g g$ ).

Results from lattice gauge theory studies suggest that the lightest gluonic hybrid mesons have $J^{P C}=1^{-+}$and mass in the range of $(1600-2000) M e V / c^{2}$. In the Fux-tube model the lightest $1-+$ isovector hybrid is predicted to decay primarily to $b_{1} \pi$ [1]. The $f_{1} \pi$ branch is also expected to be large and many other decay modes are suppressed. This suppression is consistent with recent calculations showing 1/Nc behavior for decays to spin-zero mesons in the large-Nc limit of QCD.

Few experiments have addressed the $b_{1} \pi$ and $f_{1} \pi$ meson decay channels. The VES collaboration reported a broad $J^{P C}=1^{-+}$peak in $b_{1} \pi$ decay [2], and Lee, et al. [3] observed significant $J^{P C}=1^{-+}$signal in a $f_{1} \pi$ decay. In neither case was a definitive resonance interpretation of the 1 -+ waves possible. Preliminary results from a later VES analysis show excitation of $\pi_{1}(1600)$ [4]. Significant $b_{1} \pi$ strength for $\pi_{1}(1600)$ was also reported [5]. BNL experiment E852 reported a measurement of $f_{1} \pi$ and $b_{1} \pi$ decays for $\pi_{1}(1600)$ and $\pi_{1}(2000)$ [6, 7]. In addition, claims of $J^{P C}=1^{-+}$ exotic signals exist in decay channels which were not favored by the flux-tube model. The most controversial of which is the observation of $\pi_{1}(1400) \rightarrow \eta \pi$ whereas the strongest evidence for a $J^{P C}$ exotic exists for $\pi_{1}(1600) \rightarrow \eta^{\prime} \pi$.

The $\eta \pi$ system is particularly interesting in searching for exotic (or non- $q \bar{q}$ ) mesons because the system has spin $(\mathrm{J})$, parity $(\mathrm{P})$, and charge-conjugation $(\mathrm{C})$ in sequence $J^{P C}=0^{++}, 1^{-+}, 2^{++}, 3^{-+} \ldots$ for $l=0,1,2,3, \ldots$. (Here $l$ is the orbital angular momentum of the $\eta \pi$ system.) Hence a resonance with an $\eta \pi$ decay mode with odd $l$ is manifestly exotic.

In previous publications [8, 9], Brookhaven E852 presented evidence for an exotic meson produced in the reaction $\pi^{-} p \rightarrow \eta \pi^{-} p$ at $18 \mathrm{GeV} / c$ from an analysis of the $1994 \mathrm{E} 852$ data set. A large asymmetry in the angular distribution was observed indicating interference between $l$-even and $l$ odd partial waves. The $a_{2}(1320)$ was observed in the $J^{P C}=2^{++}$wave, as was a broad enhancement between 1200 and $1600 \mathrm{MeV} / \mathrm{c}^{2}$ in the $1^{-+}$exotic wave. The observed phase difference between these waves shows that there was phase motion in addition to that due to $a_{2}(1320)$ decay. An analysis of the mass dependence of the partial waves shows that the data is well described by the interference between the $a_{2}(1320)$ and an exotic $1^{-+}$resonance with Mass $=\left(1370 \pm 16_{-30}^{+50}\right) \mathrm{MeV} / \mathrm{c}^{2}$ and $\Gamma=\left(385 \pm 40_{-105}^{+65}\right) \mathrm{MeV} / \mathrm{c}^{2}$.

E852 has performed a partial wave analysis of the reaction $\pi^{-} p \rightarrow \pi^{+} \pi^{-} \pi^{-} p$ [10]. In summary, all expected well-known states $\left(a_{1}, a_{2}\right.$, and $\left.\pi_{2}\right)$ are observed. In addition, the natural parity exchange wave with manifestly exotic quantum numbers $J^{P C}=1^{-+}$shows structure in the intensity and phase motion which is consistent with a resonance at $1600 \mathrm{MeV} / \mathrm{c}^{2}$ decaying into the $\rho \pi$ 
channel. A mass dependent fit results in a resonance mass of $1593 \pm 8_{-47}^{+29} \mathrm{MeV} / c^{2}$ and a width of $168 \pm 20_{-12}^{+150} \mathrm{MeV} / \mathrm{c}^{2}$. A recent analysis of a larger $3 \pi$ data set emphasizes discrepancies in the PWA systematics which make the claims of an exotic signal controversial [11].

A subsequent analysis studied the $\eta^{\prime} \pi^{-}$system produced in the reaction $\pi^{-} p \rightarrow \eta \pi^{+} \pi^{-} \pi^{-} p$. The data exhibit a clear signal for $\eta^{\prime} \rightarrow \eta \pi^{+} \pi^{-}$in the $\eta \pi^{+} \pi^{-}$mass spectrum. The accepted $\eta^{\prime} \pi^{-}$ mass spectrum shows a peak in the region of the $a_{2}(1320)$ and a broad peak near $1600 \mathrm{MeV} / \mathrm{c}^{2}$. The results of a partial wave analysis find two important partial waves: the $2^{++}$wave consistent with an $a_{2}(1320)$ signal and a broad higher mass structure; and a dominant $J^{P C}=1^{-+}$exotic wave which peaks at $1600 \mathrm{MeV} / \mathrm{c}^{2}$.

A study of the mass dependence of the PWA results finds three Breit-Wigner (BW) poles in the $2^{++}$wave and two BW poles in the $1^{-+}$wave. The $1^{-+}$wave is dominated by a pole at $1600 \mathrm{MeV} / c^{2}$ with a small contribution from a pole at $1400 \mathrm{MeV} / \mathrm{c}^{2}$. An alternative fit, one with a poorer $\chi^{2} / D o F$ of 1.22 , finds that the $1^{-+}$wave can be described by the pole at $1600 \mathrm{MeV} / \mathrm{c}^{2}$ only. The results for the $2^{++}$poles and the $1^{-+}$pole at $1600 \mathrm{MeV} / c^{2}$ are stable and are not affected by the parameterization of the $1^{-+}$low mass region.

Recently, g12 at Jefferson Lab's CEBAF Large Acceptance Spectrometer (CLAS) has acquired a high statistics photoproduction dataset, using a liquid hydrogen target and tagged photons from a $5.71 \mathrm{GeV}$ electron beam. The CLAS experimental apparatus was modified to maximize forward acceptance for peripheral production of mesons. The resulting data contain the worldâ largest $3 \pi$ photoproduction dataset.

The latest results, presented June 1st at the Eleventh Conference on the Intersections of Particle and Nuclear Physics (CIPANP 2012). Features of the results exhibit clear observation of the well know meson states: $a_{1}(1260), a_{2}(1320)$, and the $\pi_{2}(1670)$. The main feature is a non-observation of the $1^{-+}$exotic state in both the mass intensity and in the phase motion relative to the $p i_{2}(1670)$.

\section{The GlueX Experiment}

A recent effort at Jefferson Lab, in conjunction with the plans for the energy upgrade of CE$\mathrm{BAF}$, is the development of a state-of-the-art hermetic spectrometer-the GlueX project (formerly know as the Hall D project). One of the scientific motivations for upgrading CEBAF is a highstatistics definitive study of the photoproduction of mesons with masses below the $c \bar{c}$ threshold in a search for new forms of hadronic and gluonic matter. After many years of searching for gluonic excitation produced via hadronic probes, only now are we finding some promising candidates. An unexplored search area is the photoproduction of light quark meson states. Since the photon can be thought of as a virtual vector meson with quark spins aligned, it is a probe distinct from the traditional hadronic probes of pions, kaons, and protons. Within the Flux-tube model, the production of gluonic excitations via photon interaction is expected to produce a wealth of states with manifestly exotic $J^{P C}$ 's ( $q \bar{q}$ forbidden quantum numbers).

The goal of the GlueX experiment is a mapping of the spectrum of gluonic excitations with the ultimate objective being a quantitative understanding of the nature of confinement in QCD. To achieve this goal a hermetic detector, the GlueX spectrometer, optimized for amplitude analysis, will be constructed in a new experimental hall (Hall D). A tagger facility will produce $9 \mathrm{GeV}$ linearly polarized photons via coherent bremsstrahlung radiation of $12 \mathrm{GeV}$ electrons through a di- 


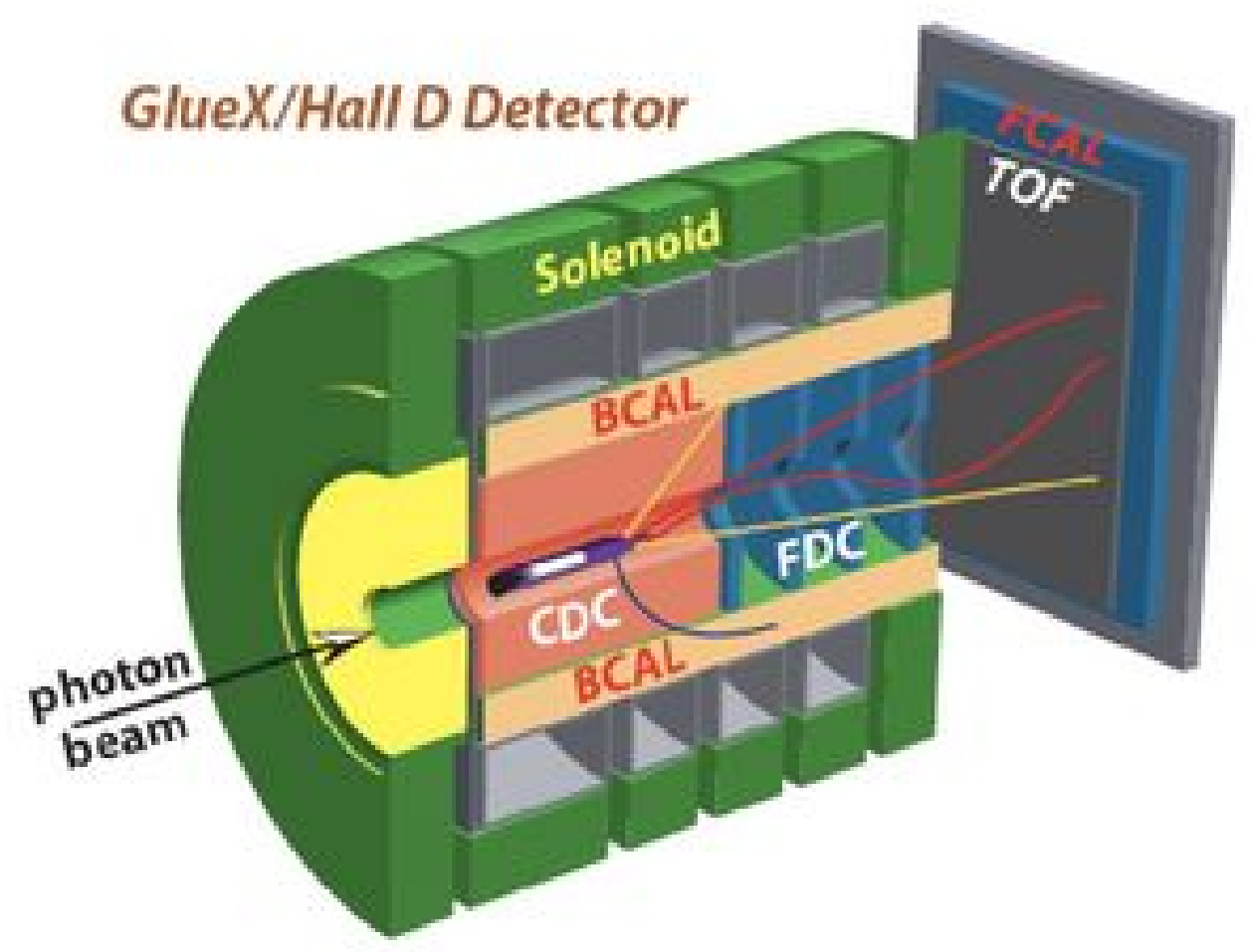

Figure 1: Schematic of the GlueX detector.

amond wafer. To achieve $12 \mathrm{GeV}$ electrons $\mathrm{CEBAF}$ will be upgraded to $12 \mathrm{GeV}$ with additional cryomodules, modified arcs and an additional arc. The GlueX detector uses a geometry based on solenoidal magnetic field (Figure 1). The superconducting solenoid produces a $2.25 \mathrm{~T}$ field. A tagged, $\approx 9 \mathrm{GeV}$, linearly polarized photon beam is incident on a $30 \mathrm{~cm}$ long liquid-hydrogen target that is surrounded by a scintillator start counter which is used in triggering. Following that is a cylindrical tracking chamber, the $\mathrm{CDC}$, and then a cylindrical electromagnetic calorimeter, the BCAL. Downstream of the CDC are four packages of circular planar drift chambers, FDC, followed by a time-of-flight wall, TOF. This is followed by a circular planar electromagnetic calorimeter, the FCAL. Space has been reserved between the downstream end of the magnet and the TOF for a future upgrade particle identification system. This design provides for nearly 4ź acceptance for both charged particles and photons. While the acceptance is not completely uniform in all variables, there are no holes in the kinematic variables of interest.

The GlueX collaboration was formed in 1998[12]. The project has been reviewed externally and by the Jefferson Lab PAC. In September 2008, the U.S. Department of Energy gave Jefferson Lab Critical Decision 3 (CD-3) approval for the CEBAF upgrade and construction of Hall D and the GlueX apparatus.

At the present time, the civil and accelerator construction are 90\% complete and the experimental equipment is 50-60\% obligated. Accelerator commissioning is planned for Jan 2014 with 
beam delivered to GlueX shortly after.

\section{References}

[1] N. Isgur and J. Paton, Phys. Rev. D31, 2910, (1985); F. E. Close and P. R. Page, Nucl. Phys. B443, 233, (1995); Philip R. Page, Phys. Rev. D70, 016004, (2004).

[2] Valery Dorofeev et al., AIP Conf. Proc. 619, 143, (2002).

[3] J.H. Lee et al., Phys. Lett. B323, 227, (1994).

[4] D.V. Amelin, et al., Yad. Fiz. 62, 487 (1999); ibid, Phys. Atom. Nucl. 62, 445, (1999).

[5] C.A. Baker et al., Phys. Lett. B563, 140, (2003).

[6] M. Lu et al. (BNL-E852 Collaboration), Phys. Rev. Lett. 94, 032002, (2005).

[7] J. Kuhn et al. (BNL-E852 Collaboration), Phys. Lett. B595, 109 (2004).

[8] D. R. Thompson et al. (BNL-E852 Collaboration), Phys. Rev. Lett. 79, 1630 (1997).

[9] S. U. Chung et al.(BNL-E852 Collaboration), Phys. Rev. D60, 072001, (1999).

[10] G. S. Adams et al. (BNL-E852 Collaboration), Phys. Rev. Lett. 81, 5760, (1998).

[11] A. R. Dzierba et al., Phys. Rev. D73, 072001, (2006).

[12] http://www.jlab.org/Hall-D, http://www.gluex.org 